\title{
La educación en ingeniería en el contexto global: propuesta para la formación de ingenieros en el primer cuarto del Siglo XXI
}

\author{
Engineering Education in the Global Context: Education Proposal \\ for the First Quarter of the 21st Century
}

\author{
Vega-González Luis Roberto \\ Coordinación de Vinculación y Gestión Tecnológica \\ Centro de Ciencias Aplicadas y Desarrollo Tecnológico (CCADET) \\ Universidad Nacional Autónoma de México \\ Correos:Irvg@servidor.unam.mx; roberto.vega@ccadet.unam.mx
}

Información del artículo: recibido: agosto de 2011, reevaluado: febrero de 2012, aceptado: agosto de 2012

\begin{abstract}
Resumen
En este trabajo se plantea que para que las facultades y escuelas en las que se forman ingenieros en México se sintonicen con la intensa dinámica de cambios, es necesario que busquen nuevas opciones de enseñanza. La experiencia reciente muestra que estas instituciones están respondiendo sólo a las necesidades inmediatas que demanda la disponibilidad de nuevas tecnologías convergentes en el sector industrial. Es urgente realizar esfuerzos permanentes de planeación en el área de educación en ingeniería, teniendo en cuenta que la educación superior debe adaptarse de la mejor manera posible a los cambios económicos y sociales. Como marco de referencia se analiza la transición que se ha dado en los últimos años en la cultura organizacional y el formato general de educación en ingeniería impartido actualmente. Dentro del marco metodológico, se analizan las acciones que están tomando algunas universidades norteamericanas, australianas y asiáticas para la enseñanza de la ingeniería. Finalmente se presenta una propuesta integrada de la forma como se podrían preparar los nuevos ingenieros para enfrentar las demandas del siglo XXI. Las ideas presentadas tendrán que ser adaptadas agregando nuevas formas y esquemas, alternativos y/o complementarios, buscando que la formación de ingenieros en México se adapte mejor y continuamente a la dinámica de cambios global.
\end{abstract}

\section{Descriptores:}

- educación

- ingeniería

- globalización

- México 


\begin{abstract}
In this paper it is suggested that in order that the faculties and engineering schools in Mexico be tuned with the intense dynamic changes, it is necessary to look for new education options. Recent experience shows that these institutions seem to be just responding to the needs of the new industrial technology platforms available. It is urgent to develop permanent planning efforts to adapt engineering education to the new economic and social world conditions. In the introduction the organizational cultural transition occurred in recent years and the actual general engineering education format are analyzed. Afterwards, North American universities engineering teaching actions are reviewed as part of the methodology. Finally, an integrated proposal about the way new engineers could be prepared to address the 21st century demands is presented. The ideas offered must be adapted and complemented to find the best schemes in order that Mexican engineer's education can adapt continuously to the global dynamic changes.
\end{abstract}

\section{Introducción}

La premisa fundamental de este trabajo es que a nivel mundial y por supuesto, también en nuestro país, los profesionales de la ingeniería siempre han estado en el centro de los procesos de innovación tecnológica, económica y social y tal vez nunca como hoy la ingeniería ha sido tan importante ante la emergencia e intensa difusión de las tecnologías de la información, robótica y de la biotecnología entre muchas otras innovaciones y descubrimientos científicos que afectan todas las áreas de la vida (Sheppard et al., 2009; Valencia, 2010).

El objetivo central es ofrecer una visión para la formación de las futuras generaciones de estudiantes y aportar elementos que puedan servir como ideas previas para el desarrollo de procesos de planeación de la educación en ingeniería. Como marco de referencia se describe en términos generales la forma como se lleva a cabo la enseñanza de la ingeniería en la actualidad y se estudian los cambios radicales que se han dado en las últimas décadas en la cultura organizacional de las empresas en las que están insertos los profesionales de esta disciplina. Considerando que la expectativa es que todas las tendencias del sistema global continuarán incrementándose intensamente en el corto y en el mediano plazo, la pregunta de fondo es, si el tipo de formación que seguimos dando en las escuelas y facultades de ingeniería es el adecuado.

La problemática se aborda metodológicamente con base en las tres dimensiones del conocimiento que reconoce la Fundación Carniegie para la formación de profesionales: el aprendizaje cognitivo, el aprendizaje práctico y el aprendizaje moral (Sheppard et al., 2009). El objeto de estudio se centra en los diferentes aspectos tanto humanos como económicos requeridos para el desarrollo de habilidades de comportamiento humano y de competencias personales, empresariales y gerenciales que además de sus conocimientos fundamentales y de aplicación deben desarrollar los futuros ingenieros con la guía y el apoyo de los profesores de las facultades y escuelas profesionales.

La intención es que los ingenieros egresados puedan insertarse correcta y oportunamente en las unidades productivas en las que les corresponderá participar para continuar siendo elementos centrales de los procesos de innovación económica y social.

De acuerdo con Igbaria et al. (1999), la importancia del planteamiento radica en que la planeación estratégica de las carreras de profesionales en investigación, desarrollo e ingeniería (RD\&E), es fundamental para el desarrollo de la economía de un país, por lo que desarrollar los mecanismos apropiados para formar y motivar adecuadamente a los profesionales de estas áreas, indudablemente emergerá en el futuro próximo como un factor crítico para el éxito de las organizaciones que quieran competir en los mercados mundiales.

Finalmente, se presenta una propuesta de cómo se podrían adaptar los procesos de formación de ingenieros para responder a la problemática tratada.

\section{El ingeniero del primer cuarto del siglo XXI}

Como marco de referencia consideramos que existen aspectos de orden individual y organizacional que debemos tomar en cuenta para llevar a cabo el ejercicio de reflexión. Para empezar, revisaremos en términos generales qué es lo que ha pasado con la cultura organizacional en las últimas décadas, entendiéndola como el conjunto de valores, creencias y principios fundamentales que compartidos por los miembros de la organización, constituyen los cimientos del sistema gerencial de la misma, así como también el conjunto de procedimien- 
tos y conductas gerenciales que sirven de ejemplo y refuerzan esos principios básicos (Denison, 1991; Alveson y Berg, 1992).

Con base en lo anterior, observemos ahora el cuadro de la figura 1, en el que según la propuesta de Gilbert y Cordey (1996), se percibe que la cultura organizacional de los directivos de las empresas en las que están insertos los diferentes tipos de trabajadores, incluyendo a los profesionistas, se ha modificado enormemente de hace unos veinte años a la fecha. La situación no es irrelevante, sino por el contrario, es extraordinariamente importante y debe estudiarse con mucho cuidado.

Desde unos doscientos años antes del advenimiento del presente siglo, dentro de las organizaciones se promovía una cultura de servicio, las empresas tenían una actitud de protección para sus empleados, por lo que se consideraba que el trabajo que las mismas ofrecían era de por vida, lo cual generaba una gran lealtad por parte de sus miembros. Se valoraba la experiencia técnica y se contaba con un sistema de promoción y aumento de salario automático. Como el cambio tecnológico era mucho más lento, el sistema permitía que aunque existieran pérdidas económicas durante un periodo de tiempo específico, la empresa tendría tiempo de recuperarse debido a la lealtad de sus clientes; es decir, en el fondo hacia el interior y hacia el exterior de las organizaciones se propiciaban los valores.

La cultura de servicio se dio en una época de mucha estabilidad, abarcando por cierto, la mayor parte del tiempo en el que ha operado la Universidad Nacional Autónoma de México, desde su fundación en 1910. De cierta manera, la cultura de servicio generaba costos inmediatos a las empresas, pero en el mediano plazo la confianza generada y la preferencia por parte de los clientes para la empresa, producía generosas utilidades garantizando su permanencia. Las empresas asumían una actitud paternalista de protección para sus empleados generando valores como la lealtad y la honradez y por lo tanto, la posibilidad de tener un trabajo de por vida.

A partir de finales del siglo pasado y particularmente de unos cuantos años a la fecha, ha habido un cambio abrupto en la cultura de servicio y recompensa a largo plazo, por la cultura de las ventas con recompensa inmediata, pero sin seguridad para los empleados. La cultura de las ventas es la cultura del interés por las utilidades inmediatas en la que las empresas están menos preocupadas por la suerte de sus empleados, quienes al tener menos seguridad nunca desarrollan valores de lealtad y pueden ser propensos a perder la honradez y a corromperse, ya que su principal motivación es la recompensa o el pago inmediato. Dado que el pago principal se realiza por desempeño, las posibilidades de promoción y de permanencia en la empresa son prácticamente nulas; sin embargo, llama la atención que no obstante la situación tan poco favorable para los empleados, las empresas cada vez requieren perfiles profesionales más altos.

Es notable que ante la situación descrita los estudiantes de cualquier carrera, y en particular los que egresan de las distintas carreras de ingeniería, se enfrenten ahora más que nunca a retos formidables.

Aunque la situación puede variar entre carreras, escuelas y universidades, veamos ahora, de manera muy general y tratando de no particularizar, cuál es la forma en la que hemos venido llevando a cabo la formación de nuestros ingenieros. En primer lugar partiremos considerando que los alumnos que ingresan a las distintas carreras de ingeniería han adquirido en su formación

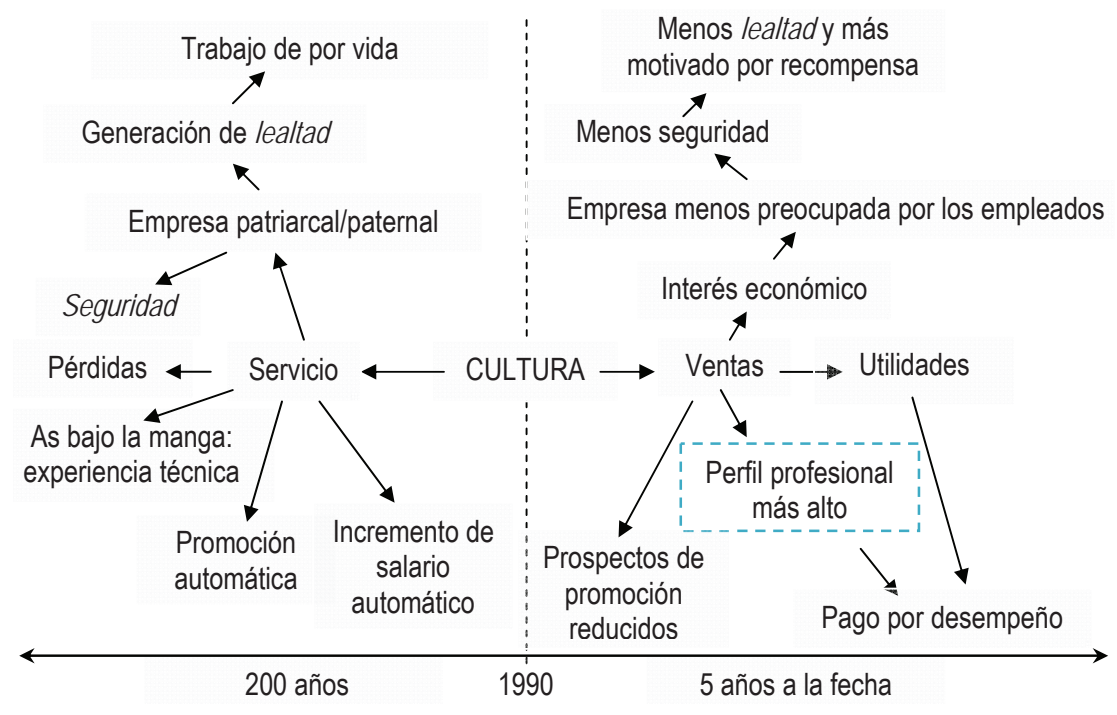

Figura 1. Resumen de la percepción de los cambios en la cultura organizacional Gilbert y Cordey (1996) 
previa un muy buen nivel de conocimientos en las materias de física y matemáticas básicas; además, deben haber adquirido un nivel mínimo de conocimientos en materias de otras áreas como la historia, geografía, español, biología y la educación cívica, entre otras. Normalmente estos temas no vuelven a cubrirse durante los estudios de ingeniería a excepción de algunas materias optativas específicas. Algunos alumnos también tienen un buen nivel de idioma extranjero.

En la parte inferior de la figura 2 podemos observar que los primeros cuatro semestres de las distintas carreras de ingeniería son dedicados a ampliar y profundizar en el conocimiento de las asignaturas correspondientes a las ciencias básicas o de conocimientos fundamentales. Últimamente se incluyó a la química como una materia relevante debido a que en muchos de los casos los ingenieros trabajan en empresas en las que se llevan a cabo procesos de transformación química para la elaboración de los productos.

Posteriormente, a este periodo de preparación inicial se llevan a cabo los semestres de formación de los alumnos en los que se imparten asignaturas de ciencias de la ingeniería, en un periodo que en promedio oscila entre dos y cuatro semestres; es decir, del cuarto al sexto $\mathrm{u}$ octavo semestre. Durante este tiempo, las y los alumnos deberán llevar las materias que serán la columna vertebral de su carrera de acuerdo con el plan de estudios correspondiente que se encuentre vigente y aprobado por el consejo técnico de la facultad o la escuela correspondiente.

El número y tipo de asignaturas depende de cada carrera; así, mientras que un ingeniero en minas y metalurgista se enfoca en materias tales como procesos de refinación de minerales, metalurgias, cristalografía de minerales, etcétera; un ingeniero petrolero lleva materias relacionadas a la exploración y refinación del petróleo, un ingeniero en telecomunicaciones trabaja entre otros, en los siguientes temas: teoría electromagnética, antenas y microondas; un ingeniero en mecánica aprende diseño de mecanismos y procesos de manufactura, un ingeniero en sistemas eléctricos de potencia cubre materias como máquinas eléctricas y turbomaquinaria, entre muchas otras. Un ingeniero industrial hace énfasis en ingeniería económica y financiera, en investigación de operaciones,

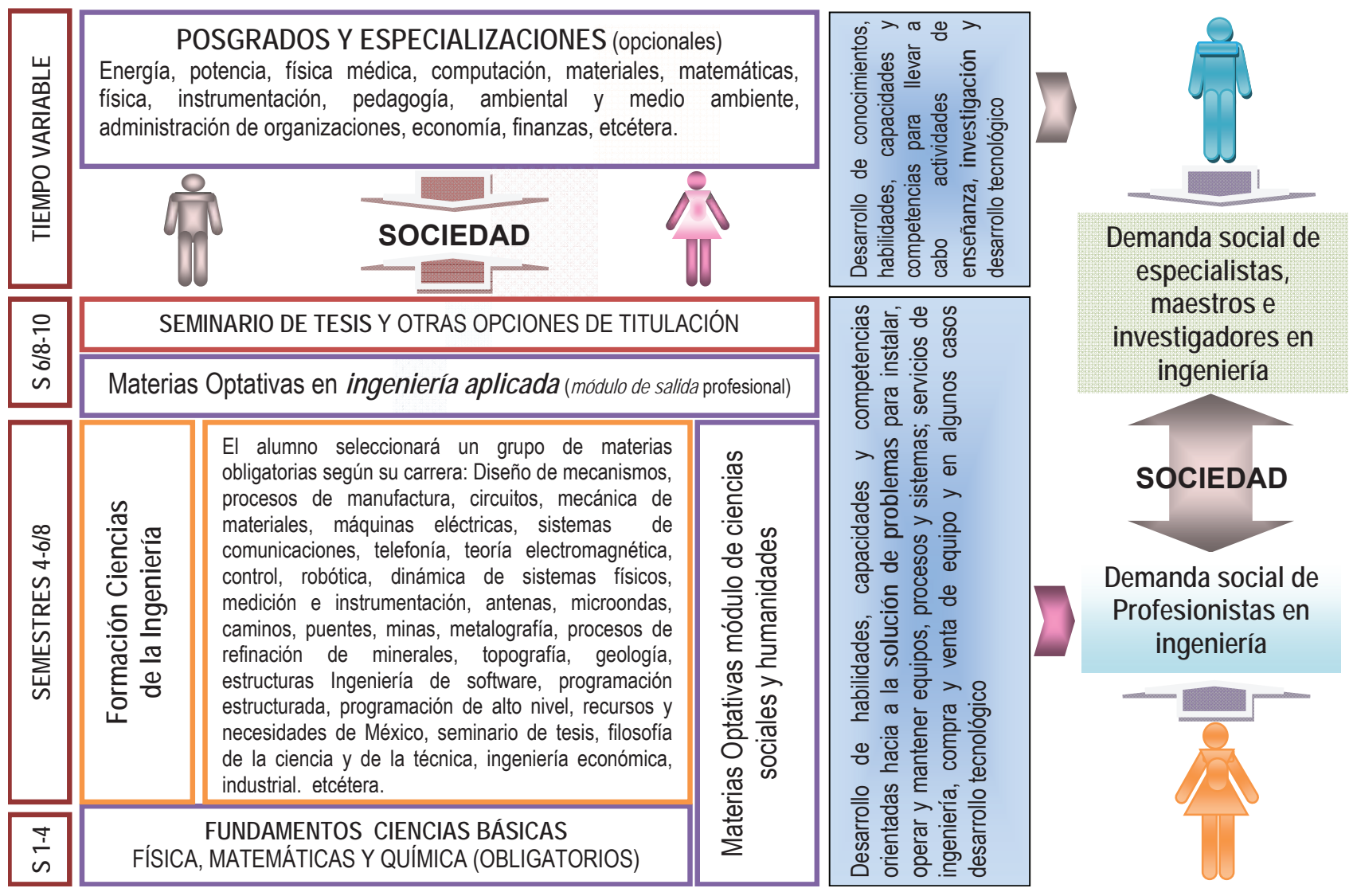

Figura 2. Esquema de formación actual que por lo general se sigue en las carreras de ingeniería (Elaboración propia) 
el ingeniero en mecatrónica cubre materias relativas a mecánica de precisión, micro dispositivos, sistemas electrónicos y así sucesivamente.

La última parte de la formación básica está conformada por el módulo de asignaturas en ingeniería aplicada obligatorias que definirá el alumno como su módulo de salida profesional. El tiempo típico para esta parte final de la carrera es de dos a tres semestres. En muchos casos, desde el principio de la carrera el alumno puede complementar su formación a través de una serie de materias optativas en las áreas de humanidades que cubren aspectos culturales, económicos o literarios.

Los tiempos y la forma de llevar a cabo la carrera pueden variar y dependen fundamentalmente del plan de estudios y del trabajo que desarrolle cada alumno.

Aunque en algunas escuelas o facultades que ofrecen carreras de ingeniería no se requiere alguna forma de titulación, en la mayoría de los casos, cuando el alumno termina con $100 \%$ de sus créditos debe optar por alguna de las diversas opciones de titulación disponibles. Las más comunes son: la tesis, la presentación de una tesina con informe de trabajo profesional, el examen general de conocimientos, o la acreditación de estudios cuando el alumno continúa realizando estudios de maestría directamente. Después de salir de su carrera, aquellos alumnos con interés en ampliar aún más sus conocimientos y mejorar sus habilidades en forma sustantiva en algún área particular del conocimiento, pueden estudiar especializaciones profesionalizantes para su aplicación inmediata.

Por otra parte, si el alumno encuentra una vocación especial para la investigación o la docencia, cuenta con la posibilidad de realizar estudios de maestría y posteriormente doctorado.

Para complementar el marco de referencia, a continuación revisaremos qué está sucediendo y cuáles son las acciones que están siguiendo las escuelas de ingeniería de algunas universidades importantes de diversos países. De esta manera observaremos que las funciones fundamentales del ingeniero ya no pueden sólo seguir siendo orientadas hacia la asimilación de tecnologías para resolver problemas relativos a la instalación, operación y el mantenimiento de los sistemas productivos.

\section{Aspectos relevantes de los ingenieros del siglo XXI}

Desarrollo de habilidades de comportamiento humano

A nivel internacional se ha reconocido la necesidad de incorporar habilidades de comportamiento humano en la educación de ingeniería con el fin de obtener una educación balanceada entre atributos técnicos y no técnicos (Hsu, 2004; Cordoba, 2007).

Según el Consejo para la Acreditación para Ingeniería y Tecnología (ABET), en el mercado de trabajo se espera que los ingenieros sean capaces de hablar, interactuar y trabajar con gente de diferente formación, que sean capaces de transformarse en líderes si la situación es adecuada, que sean éticos y que se conduzcan efectivamente en los ambientes profesionales, por lo que ha propuesto que las siguientes habilidades de comportamiento humano son necesarias para que los nuevos ingenieros puedan desenvolverse mejor en su trabajo: habilidades de comunicación, trabajo en equipo, negociación, relaciones interpersonales, administración, ética, aprendizaje de por vida, inteligencia emocional y creatividad (ABET, 2004; Selinger, 2003; Hissey, 2000; Nguyen, 1998). A continuación se describen dichas habilidades.

\section{Comunicación}

Dado que la comunicación ocurre cuando las ideas de cada parte son entendidas con precisión, poseer habilidades para la comunicación oral y escrita es extraordinariamente importante y es un asunto clave para el éxito en el trabajo y en todos los aspectos de la vida. Se estima que en la práctica profesional los ingenieros dedican entre un 50 a un $75 \%$ de su tiempo a aspectos de comunicación (Baren y Watson, 1991). Para los ingenieros es fundamental desarrollar habilidades para definir problemas, soluciones y resultados, establecer procesos de negociación, participar en sesiones de trabajo, juntas de grupo y seminarios para transferir y asimilar tecnología, traducir la información técnica a una forma simple y clara de entender aunque sea numéricamente rica (Darling y Daneels, 2003). Según considera Robar (1998), aquellos ingenieros que se comunican bien tienen más posibilidades de éxito profesional, que quienes no lo hacen, independientemente de su experiencia técnica.

\section{Trabajo en equipo}

Actualmente, el ambiente de trabajo de los ingenieros está orientado a la formación de equipos de trabajo (team oriented environments), el desarrollo de proyectos requiere del establecimiento de grupos formados porgente de diversos departamentos funcionales de las organizaciones (Sundstrom et al., 1990). Por lo tanto, para tener éxito en una empresa o en cualquier otro ambiente de trabajo profesional, los ingenieros deben prepa- 
rarse para trabajar en equipos diversos y multifuncionales. Para que los equipos de trabajo operen adecuadamente, es muy importante que se hagan esfuerzos para asegurar que los integrantes de los mismos reciban el conocimiento y las herramientas necesarias (Lindgard y Berry, 2002). Entre otros aspectos, los miembros de los equipos deben estar de acuerdo en las metas, problemas y soluciones planteadas para el proyecto; comunicarse efectivamente, apoyarse y tener confianza mutua, entrenarse y asesorarse entre ellos, escucharse y respetarse, tener orgullo y gozar de la actividad del grupo, así como tener una orientación fuertemente orientada a las normas de desempeño y a los resultados.

El desempeño de los equipos de desarrollo ha sido explicado por Scott (1997), en términos de la identificación social de sus miembros, lograda a través de los procesos de comunicación y reconocimiento de sus líderes, lo cual favorece la cohesividad entre ellos. Los equipos de trabajo han sido llamados por Brown y Eisenhart (1995; citados por Scott, 1997), "el corazón del desarrollo eficiente de nuevos productos".

\section{Habilidades interpersonales}

En este apartado se incluyen las habilidades de saber leer y manejar las emociones, así como las motivaciones y los comportamientos propios y ajenos durante la interacción social o en los contextos en los que hay gran interactividad social. Desarrollar habilidades interpersonales permite a los ingenieros construir consensos, manejar conflictos en forma efectiva, tener la capacidad de trabajar con otros y tener buen entendimiento con ellos, manejar grupos, reconocer las fortalezas y habilidades de otros, usar estrategias de comunicación y persuasión efectivas; saber escuchar, entender y manejar apropiadamente las emociones de otros y manejar su propio comportamiento durante las interacciones sociales. Según Simmons (1999), las habilidades interpersonales jugarán un papel muy importante durante la carrera del ingeniero, del gerente de ingeniería, del gerente organizacional y del ejecutivo; sin embargo, un débil entendimiento y la incapacidad de aplicar habilidades interpersonales puede limitar seriamente su carrera.

\section{Inteligencia emocional}

Se refiere a la capacidad para reconocer nuestros propios sentimientos y los de los otros a fin de motivarnos y manejar bien nuestras emociones y relaciones. Esto se hace relevante en una época en la que los proyectos de ingeniería son multidisciplinarios por necesidad y en muchos casos globales. Por lo tanto, en el ambiente en el que el ingeniero moderno tiene que interactuar se encuentran personas con otras profesiones y de otras culturas. Según Goleman (1999), los dominios de la inteligencia emocional son la auto-confianza, el autocontrol, la motivación, la empatía y las habilidades de socialización. Debemos reconocer que hoy en día es indispensable incorporar estas áreas en los programas de estudio de los nuevos ingenieros, para prepararlos para su vida profesional.

Todo parece indicar que la capacidad intelectual del nuevo profesionista está directamente relacionada con su dominio del conocimiento técnico y tecnológico de su área de conocimiento, lo cual favorecerá su contratación; mientras, que su inteligencia emocional le permitirá ser promovido y debido a las condiciones actuales de la economía mantener su empleo.

Cuando un individuo desarrolla su inteligencia emocional crece en los siguientes aspectos:

a) tiene confianza en sí mismo y tiende a delegar trabajo a otros y los motiva para dar lo mejor de sí mismos,

b) por ser buen administrador de sus propias cualidades tiene actitud proactiva y toma acciones anticipadas para prevenir problemas, pero también saca provecho de las oportunidades disponibles,

c) tiene buena penetración social, escucha y se preocupa por lo que la gente le dice, tiene empatía respecto a otras personas (Sunindijo y Hadikisumo, 2005).

Es fundamental desarrollar la personalidad de los estudiantes de ingeniería a través de la inteligencia emocional para eliminar la imagen estereotipada negativa del ingeniero como un genio socialmente inadaptado, lo cual muchas veces impide su reclutamiento y promoción (Yurtseven, 2001; Clarke, 2010).

\section{Ética y autonomía moral}

Como todas las disciplinas de aprendizaje modernas, la ingeniería debe hacerse preguntas acerca del significado social y los efectos del conocimiento que generan (Jennings, 2010).

La preocupación del comportamiento ético ha estado relacionada con las actividades tecnológicas desde hace muchos años. Desde 1940, la ABET, que entonces era el Consejo para el Desarrollo Profesional de la Ingeniería (ECPD), mostró interés en la introducción de educación ética en los programas de ingeniería formando un Comité en Principios de Ética en la Ingeniería. Hoy en día se ha establecido como una disciplina reco- 
nocida. Según Herkert (2000), en el último cuarto del siglo XX en los Estados Unidos de Norteamérica se realizaron cambios notables en la educación en ingeniería, entre los cuales destacan el reconocimiento a la ética, la moral y la responsabilidad social.

Indiscutiblemente, el trabajo del ingeniero tiene un impacto significativo en la sociedad, por lo tanto, la práctica de la ingeniería acarrea obligaciones y responsabilidades. Los ingenieros deben reconocer tanto los impactos positivos como los negativos de las soluciones particulares de ingeniería que recomienden. También deben informar a la sociedad de esos impactos a fin de obtener un consentimiento informado antes de que las acciones ingenieriles se lleven a cabo. Los ingenieros deben actuar éticamente para reconocer y resolver conflictos potenciales con otros miembros de la sociedad tales como los patrocinadores, sus empleadores, los trabajadores o inclusive con otros ingenieros. Stephan (2002) sugiere que en el mundo de la ingeniería del futuro, entender la práctica y la teoría de la ética en la ingeniería será tan necesario como lo es hoy el conocimiento de las ecuaciones diferenciales, para una apropiada educación de los ingenieros.

Para actuar éticamente no sólo se requiere la intuición, sino que es necesario que el alumno estudie los fundamentos y adquiera habilidades éticas en el salón de clase para que pueda entender y manejar asuntos en los que se necesite la toma de decisiones responsable, de esta manera se impulsa la autonomía moral de los futuros ingenieros (Marshall y Marshall, 2007). Al obtener fundamentos y habilidades éticas, los nuevos ingenieros obtendrán confianza para identificar y razonar acerca de problemas morales, evaluar las situaciones con criterio moral, actuar moralmente y distinguir entre situaciones éticas y no éticas, entender las consecuencias de sus acciones y su responsabilidad hacia la sociedad (Loui, 2004; citado por Cordoba, 2007).

\section{Desarrollo continuo de capacidades y competen- cias personales, empresariales y gerenciales}

Debido al papel tan importante que tienen las empresas en los procesos de innovación tecnológica, económica y social, actualmente es fundamental apoyar a los estudiantes de ingeniería con el aprendizaje de otros aspectos y competencias para el desarrollo empresarial y gerencial, tales como los que describiremos a continuación.

\section{Aprendizaje de por vida en ingeniería}

El concepto de que la ingeniería es una disciplina estrechamente ligada con la tecnología define en sí mismo el interés y la vocación de los ingenieros; sin embargo, debido a que en las últimas décadas se ha dado una intensa dinámica de cambio tecnológico, el ciclo de vida de las tecnologías se ha venido acortando, por lo que la profesión de ingeniería se ha visto sujeta a innovación constante ya que si bien es cierto que los conocimientos básicos tienen mayor permanencia, se estima que el conocimiento de ingeniería de aplicación que adquieren los ingenieros durante sus últimos años de estudio, se hace obsoleto en un tiempo que va de entre tres a cinco años. Algunos de los efectos de esta situación en la vida profesional son el cambio continuo de empleos, la pérdida del mismo y el acortamiento del rango de vida útil en el que hay interés por los servicios de los ingenieros, de parte de quienes los emplean.

Por lo tanto, para mantener su ventaja competitiva los ingenieros deben responder muy rápido a las presiones del mercado global tratando siempre de ir un paso adelante en la incorporación de nuevo conocimiento a sus productos. Entonces, para mantenerse efectivos profesionalmente, mantener sus trabajos y avanzar en sus carreras, los ingenieros deben actualizar permanentemente sus conocimientos a través de su vida profesional (Zhang et al., 2011).

\section{Creatividad}

La creatividad se define como la habilidad de producir trabajo original y novedoso, de alta calidad y apropiado. En ese sentido, la ingeniería es la aplicación creativa de los principios de la ciencia básica y la expresión tecnológica de la ciencia aplicada. La ingeniería requiere innovación y creatividad y se enfoca en el diseño de sistemas y procesos, por lo que dado que el diseño es el corazón de la ingeniería, es en este campo donde los ingenieros profesionales demuestran su creatividad y su innovación; sin embargo, aunque es un lugar común $\mathrm{y}$ los ingenieros saben que hay muchas formas de resolver un problema y muchos caminos para llegar a una solución, no se comunica en forma explícita a los estudiantes el valor de la creatividad y por lo general no se toma como una prioridad en su educación.

La creatividad es esencial para desarrollar proyectos de investigación, desarrollo e ingeniería, según Kratzer et al. (2008), está estrechamente ligada con el papel de los líderes de los grupos de trabajo, quienes actuando con un rol central en la periferia del sistema de comunicación de los grupos, estimulan la creatividad cuando actúan monitoreando el entorno y convirtiéndose en fuentes externas o proveedores de información, noticias técnicas y nuevo conocimiento para el resto del equipo de trabajo. 


\section{Liderazgo}

Según Prahalad (Ignatius, 2010), el liderazgo, tiene que ver con la auto-conciencia personal, el reconocimiento de las fallas propias, el desarrollo de la modestia, de la humildad y de la humanidad; por lo tanto, puede describirse como la habilidad para influenciar en otras personas que siguen al individuo líder y le permiten obtener resultados significativos. También incluye la capacidad de tomar decisiones y asistir a los miembros del equipo y subordinados con la idea de maximizar el uso de recursos para obtener un objetivo común. Liderar es acompañar a otros para su desarrollo y compartir experiencias, conocimiento y visión. El liderazgo ocurre en todos los niveles de una organización y no sólo es una destreza comúnmente asignada a una sola persona que se encuentra en una posición de poder (Crawford, 1998; citado por Cordoba, 2008).

Por lo tanto, es muy importante apoyar a los estudiantes de ingeniería para que desarrollen habilidades de liderazgo y contribuyan encabezando el cambio organizacional necesario para el desarrollo de proyectos de ingeniería multidisciplinarios, construir buenos equipos, motivar a la gente, crear estructuras organizacionales innovadoras a la vez que se resuelven problemas mediante el desarrollo de trabajo efectivo. Askew y Price (2003) sugieren que los programas de ingeniería de las universidades deben proveer a los estudiantes oportunidades para desarrollar estas habilidades.

\section{Habilidades gerenciales}

Debido a que en el mundo las organizaciones están disminuyendo su tamaño a través de reestructuras y reingeniería, y aunque tradicionalmente la ingeniería y la administración se han percibido como disciplinas separadas, el nuevo ingeniero requiere adquirir habilidades gerenciales para competir. La idea no es pensar que todos los ingenieros deben convertirse en gerentes, sino darles elementos de progreso para su carrera. Sus probabilidades de éxito aumentarán si además de un sólido conocimiento técnico de su disciplina, los ingenieros obtienen un dominio mínimo de habilidades gerenciales. Para la ABET (2004), el criterio es que las habilidades gerenciales deben desarrollarse desde los inicios de la carrera, hasta los cursos finales de la misma. El principio básico se deriva de que la mayoría de los profesionales de ingeniería experimentados piensan que durante la vida profesional del ingeniero, toma más tiempo la solución de problemas en los que se requiere manejar personal, que el requerido en la solución de problemas técnicos. Las habilidades administrativas también permiten, entre otras cosas, integrar los esfuerzos multidisciplinarios, planear y lograr acuerdos, realizar comunicación efectiva, así como colectar y filtrar la información que sea más relevante para la toma de decisiones.

\section{Desarrollo de nuevos productos y emprendimiento}

Dehesa (2008), propone que existen tres formas principales para lograr el crecimiento de cualquier economía:

a) la acumulación de trabajo y capital,

b) el desarrollo del comercio y de las ventajas comparativas y

c) el conocimiento, la innovación y el emprendimiento.

En los países desarrollados el conocimiento, las ideas, las invenciones y las empresas provienen principalmente de las universidades y de las instituciones de investigación y desarrollo; por ejemplo, los académicos y los estudiantes del Instituto Tecnológico de Massachussets (MIT) producen en promedio dos invenciones diariamente y han generado más de 5000 empresas alrededor del mundo (Preston, 2001; citado por de la Dehesa, 2008). Sin embargo, en el caso de los países subdesarrollados los impulsores de la economía son la acumulación del trabajo y del capital, y en el caso de los países en vías de desarrollo se hacen intentos de buscar ventajas comparativas y de desarrollar empresas e innovaciones aplicando conocimiento.

En México vislumbramos un panorama poco alentador, las grandes corporaciones han desaparecido o han disminuido su tamaño provocando que la tasa de desempleo siga en ascenso, llegando a $5.7 \%$ de la población económicamente activa (PEA) durante algunos meses de 2010 y manteniéndose arriba de $5 \%$ de la PEA durante todo el año siguiente (INEGI, 2011, Indexmundi, 2011); dado que cada vez existen menos corporaciones que ofrezcan empleos, es imperativo tomar el ejemplo de la mayoría de los países en vías de desarrollo y formar ingenieros emprendedores promoviendo el desarrollo de talentos gerenciales y la actitud de emprendimiento en los estudiantes de ingeniería, para que ellos mismos generen sus propias fuentes de trabajo.

Es indispensable que a lo largo de su carrera, los estudiantes adquieran las herramientas indispensables que les permitan iniciar y hacer crecer sus propios negocios. Por esta razón deben aprender habilidades de administración efectivas. Los ingenieros deberán ser líderes potenciales no sólo ofreciendo servicios de ingeniería tales como la venta, la instalación y el mantenimiento de equipos y sistemas, sino que también debe- 
rán encontrar formas de producir nuevos productos que generen utilidades para satisfacer a sus clientes $\mathrm{Sin}$ duda, para encontrar nuevas soluciones a través de dispositivos que favorezcan la creación de empresas, el ingeniero del presente y del futuro inmediato deberá estar involucrado y familiarizado con la ejecución de proyectos relativos con el desarrollo de nuevos productos tecnológicos.

\section{Propuesta de un nuevo esquema para la forma- ción de los ingenieros del Siglo XXI}

En la figura 3 se muestra una comparación entre la forma tradicional de enseñanza y la propuesta que estamos planteando para la formación de los nuevos ingenieros. En ambos casos, se considera indispensable que la formación de los profesionales en ingeniería, debe partir del aprendizaje de los fundamentos físicomatemáticos y de las materias de formación en ciencias de la ingeniería que constituirán la espina dorsal de la carrera elegida por el estudiante. En la parte alta y a la izquierda de la figura, podemos observar que en el sistema de formación tradicional, los alumnos pueden optar por asociarse en algún proyecto de investigación o académico bajo la tutela de un profesor o investigador, de tal manera que podrá optar por titularse a través de la realización de una tesis o, en el caso de algunas escuelas de ingeniería, por medio de alguna de las opciones de titulación existentes. Una de las características principales de los alumnos que así se forman, es que, normalmente están orientados a resolver los problemas que les plantea su profesor o su tutor de tesis.

Una vez que los alumnos salen de la licenciatura tienen al menos tres opciones:

a) optar por seguir estudiando en el posgrado de su preferencia para realizar alguna especialización o maestría,

b) buscar trabajo en alguna empresa con lo cual ingresan al mercado de trabajo profesional y participan en la satisfacción de la demanda social de los profesionales de ingeniería; o bien,

c) trabajar en alguna empresa y estudiar en tiempo parcial.

En la parte baja y a la izquierda de la figura 3, podemos observar que la parte principal de la nueva propuesta para la formación de ingenieros es que a diferencia del

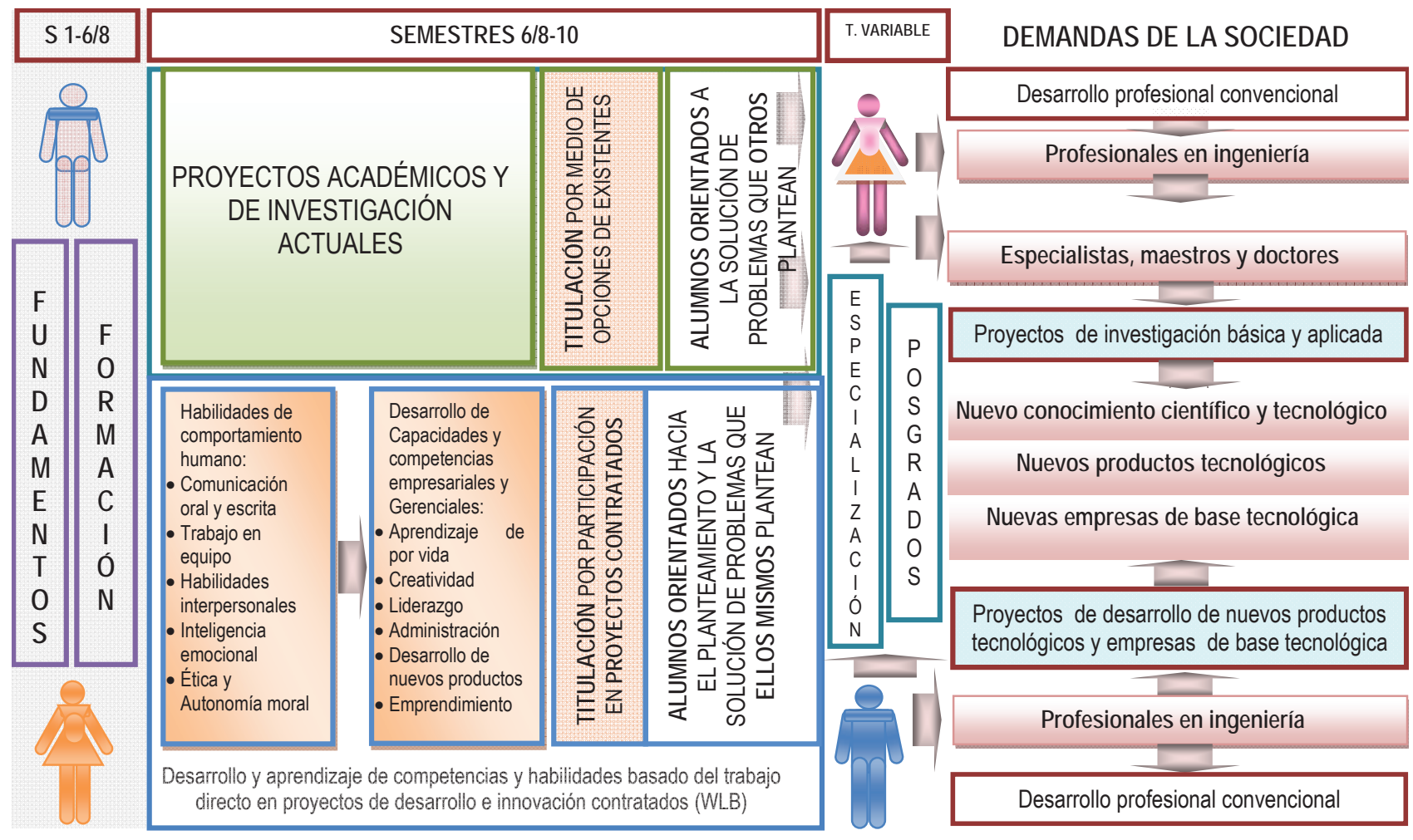

Figura 3. Propuesta para la nueva formación de ingenieros (elaboración propia) 
esquema de formación tradicional, los alumnos puedan enlistarse en proyectos de desarrollo tecnológico o innovación contratados por la universidad con empresas u organizaciones externas, en el contexto del sistema de aprendizaje por trabajo (WBL), propuesto por Inceoglu y Shukla (2011). El esquema propuesto podría entenderse como una opción terminal para las carreras de ingeniería a través de un Seminario de Proyectos de Desarrollo Tecnológico e Innovación. La idea no es nueva, ya que se promueve desde hace algunas décadas en los países desarrollados.

Por ejemplo, en los Estados Unidos de Norteamérica los programas de ingeniería de muchas universidades, auspiciados por la ABET y la Fundación Nacional para la Ciencia (NSF, por sus siglas en inglés), promueven el trabajo en equipo integrando estudiantes en sus proyectos en curso. Según Grigg (1994), el Consejo de Educación Superior de Australia ha propuesto que las Universidades deben aumentar sus actividades empresariales y sus relaciones con la industria, obteniendo fondos externos que les permitan lograr desarrollos novedosos, los cuales no serían posibles con los fondos públicos.

En el sistema de formación tradicional que se lleva a cabo en las universidades mexicanas, son muy pocos los alumnos que tienen la oportunidad de integrarse a proyectos contratados por empresas $\mathrm{u}$ organizaciones externas, en los cuales el resultado tecnológico normalmente tiene un enfoque comercial o de innovación.

Algunas ideas básicas del nuevo sistema de formación propuesto son:

a) a diferencia del esquema de formación tradicional, los proyectos desarrollados deberán estar enfocados a resolver necesidades de las organizaciones o clientes contratantes, por lo tanto, el foco del estudio es la aplicación de los conocimientos adquiridos en la universidad en un contexto de trabajo en el mundo real,

b) se requiere que los estudiantes que se integren al programa tengan un alto nivel de compromiso dentro y fuera del salón de clase, lo que permitirá obtener un buen rango de resultados medibles,

c) al participar en proyectos de desarrollo e innovación contratados por el cliente (customer driven), el alumno experimentará un tipo de aprendizaje muy flexible y adquirirá una visión más amplia de cuál es el problema que el contratante pretende resolver en el contexto económico y tecnológico de su organización, así como un panorama de cuáles son los diferentes aspectos y objetivos que persigue al financiar o participar parcialmente en el financiamiento requerido para el desarrollo del proyecto (Hauser $\mathrm{Y}$ Zettelmeyer, 1997).
Se propone como condición fundamental, que el alumno lleve cursos formales o en una primera etapa, inclusive talleres, que le permitan el desarrollo de las habilidades de comportamiento humano que hemos descrito previamente: comunicación oral y escrita, trabajo en equipo, habilidades interpersonales, inteligencia emocional, ética y autonomía moral. Como hemos visto, el desarrollo de las habilidades de comportamiento humano está estrechamente ligado al desarrollo de competencias empresariales y gerenciales, por lo que una primera posibilidad es que las actividades de aprendizaje para el desarrollo de estas competencias se lleven a cabo posteriormente.

Los cursos o talleres deberán ser conducidos por profesores o especialistas y deberán llevarse a cabo mientras el alumno realiza su trabajo en el proyecto de desarrollo e innovación del cual forma parte. Para poder implementar este nuevo sistema formativo, es muy posible que previamente se requiera generar equipos de trabajo y entrenar y capacitar a los profesores que formarán parte del mismo.

Siguiendo este sistema los alumnos podrán titularse presentando el informe de su participación en los proyectos de adscripción, en donde deberán hacer énfasis en cuál fue su aportación para el desarrollo del proyecto. La diferencia sustancial de este sistema formativo propuesto es que debido a que los alumnos que se titulen de esta forma, llevaron cursos de formación para el desarrollo de nuevos productos, su administración y emprendimiento, estarán más orientados hacia el planteamiento y solución de problemas que ellos mismos se planteen y no solamente hacia la solución de problemas de ingeniería planteados por su tutor o sus futuros contratantes.

El egresado con este perfil también podrá optar por continuar su educación inscribiéndose directamente a la especialización o la maestría, o bien, contratarse con alguna empresa u organización contribuyendo a satisfacer la demanda de profesionales de ingeniería que requiere la sociedad. Sin embargo, el ingeniero del Siglo XXI que reciba el nuevo esquema de formación propuesto también estará capacitado para llevar a cabo proyectos de desarrollo de nuevos productos tecnológicos y tendrá los elementos que le permitan participar en la formación de las nuevas empresas de base tecnológica que son requeridas urgentemente por la sociedad.

A nivel mundial las universidades se dirigen al desarrollo de programas para la enseñanza de la innovación, pero debe quedar claro que no se trata de tomar como objeto de estudio los fenómenos y procesos relacionados con la innovación, sino de enseñar a los futuros ingenieros cómo participar tomando parte activa en los procesos de la misma, en los que el papel de la inge- 
niería es fundamental, por lo que para lograrlo proponemos involucrar a los alumnos en proyectos con demanda y patrocinio externo.

Por otra parte, los alumnos de cualquiera de los esquemas de formación de licenciatura presentados que egresen de especializaciones, maestrías o doctorados, podrán por un lado satisfacer la demanda de posgraduados que tiene la sociedad y por el otro estarán capacitados para desarrollar proyectos de investigación básica y aplicada, aportando nuevo conocimiento científico y tecnológico a la sociedad, adicionalmente podrán participar en los proyectos de desarrollo de nuevos productos tecnológicos.

\section{Discusión}

Debemos aprovechar que aunque nuestro país todavía se encuentra en etapas tempranas de desarrollo económico, cuenta con el capital intelectual de los nuevos ingenieros y otros profesionistas, quienes a través de su creatividad y su talento tienen un gran potencial para realizar progresos tecnológicos muy rápidamente. Según Kondo (2005), la estrategia tecnológica debe estar alineada a la forma en la que el país se desarrolla; es decir, con su estrategia de desarrollo. A su vez, la estrategia tecnológica está muy relacionada con las estrategias formativas de sus tecnólogos, por lo que es de suma importancia realizar los ajustes que sean necesarios en las carreras de ingeniería para que los ingenieros sigan siendo los profesionistas requeridos por la nueva sociedad basada en la economía del conocimiento.

Hasta ahora las estrategias seguidas en términos de formación de recursos humanos que han venido ofreciendo las facultades y escuelas de ingeniería, incluyendo el caso de los profesionales de otras especialidades que participan en el funcionamiento y en el desarrollo de nuevas empresas, son la posibilidad de realizar estudios de maestría en administración de organizaciones, en finanzas o en contaduría, entre otras. También se ofrecen diplomados o cursos de educación continua; sin embargo, la diferencia fundamental con el esquema de formación propuesto, es que éste considera que tanto los cursos formales o talleres para el desarrollo de habilidades humanas y el desarrollo de capacidades gerenciales, deberán llevarse a cabo dentro del programa de los estudios de la licenciatura.

Antes de que se lleven a cabo los ejercicios de planeación que sean requeridos entre los diferentes consejos académicos para llegar a los acuerdos sobre las acciones pertinentes en el ámbito de los reglamentos de vigentes en las diferentes instituciones, es primordial que los grupos académicos lleguen al convencimiento de las bondades de ajustar la curricula de estudios de los nuevos ingenieros ofreciendo cursos formales o talleres para el desarrollo de habilidades humanas, las cuales a su vez, influirán en el desarrollo de capacidades gerenciales, todo esto aunado al trabajo de los alumnos en proyectos contratados para desarrollar tecnología, promoviendo la innovación dentro de un ambiente empresarial en el que el que se espera que el nuevo ingeniero realice todas sus acciones y decisiones en un marco de ética y autonomía moral.

\section{Conclusiones}

Uno de los objetivos de la planeación es identificar las acciones que permitan crear el futuro deseado a partir de las condiciones presentes en cualquier sistema. En el Plan Nacional de Desarrollo 2007-2012 se sustenta en buena medida el futuro que requieren los mexicanos respecto a las instituciones académicas hacia el año 2030 (SEP, 2009).

Con las ideas presentadas en este trabajo se pretende contribuir en los trabajos de planeación para crear el futuro que será no sólo deseado sino requerido para que la formación de los nuevos ingenieros esté acorde a la intensa dinámica de los cambios tecnológicos y sociales.

El primer aspecto a considerar es que en la actualidad es necesario implementar los mecanismos para que la universidad forme ingenieros con vocación innovadora y emprendedora, los cuales contribuyan con el desarrollo de nuevas tecnologías limpias, apropiadas y generen empresas autosustentables para mejorar el bienestar de la sociedad. La orientación de la formación de los nuevos ingenieros debe ampliarse y complementarse en los últimos semestres de su carrera, reconociendo que la tecnología es una máquina de crecimiento y el papel fundamental de los ingenieros en el desarrollo tecnológico.

Un segundo aspecto es que de acuerdo con Hecker (1997) y Zhang et al. (2011) el alumno debe entender que el aprendizaje de los ingenieros tiene que ser permanente y no pensar que la educación termina con la escuela, sino que es un proyecto de por vida, y que además es responsabilidad de quienes los emplean y quienes los educan, insistir, promover y hacerles ver la necesidad de mantenerse en la ruta del aprendizaje por el resto de su vida profesional. Una buena forma de combatir la obsolescencia técnica es a través de actualización continua (Aryee, 1991).

Otro punto relevante es que para aumentar las posibilidades de que los ingenieros egresados tengan una pronta inserción en los sistemas productivos, las nuevas generaciones se deben preparar para el mercado de 
trabajo global, incrementando la movilidad de los estudiantes y de los alumnos graduados y promoviendo que realicen estancias en instituciones del extranjero (Widding y Lohmann, 2007).

De manera que también resulta indispensable impulsar el manejo de idiomas extranjeros como un asunto crítico y de alta prioridad, para crear redes de conocimiento internacionales sostenibles. Además, es importante impulsar que los miembros de las facultades obtengan diplomas de maestrías y doctorados en el extranjero. En forma complementaria, en muchas escuelas de ingeniería se contratan profesores extranjeros con la intención de que los alumnos de ingeniería se involucren en los proyectos de I\&D y en las redes de innovación internacionales en las cuales estos profesores participen o sean responsables.

Las acciones señaladas complementarán al nuevo sistema de formación de ingenieros propuesto, ya que el desarrollo de habilidades interpersonales apoyará y facilitará la movilidad internacional de los estudiantes.

Es necesario actuar de inmediato ya que si se logra la inclusión del sistema formativo comentado a lo largo de este trabajo, los resultados del mismo en términos de su impacto social posiblemente se verán hasta la siguiente década debido a la enorme inercia del sistema social y en particular del subsistema de educación superior.

\section{Referencias}

ABET (2004). Sustaining the Change: A Follow-up Report to the Vision for Change [en línea] [fecha de consulta: el 8 de enero de 2011]. Disponible en: http://www.abet.org/papers.shtml.

Alveson M., Berg P.O. Corporate Culture and Organizational Symbolism. Walter de Gruyter \& Co. Berlin, p. 258, 1992.

Aryee S. Combating Obsolescence: Predictors of Technical Updating Among Engineers. Journal of Engineering Technology $\mathcal{E}$ Management, volumen 8, 1191: 103-119,

Askew P.E., Price R.L. Advancing Student Leadership Development. Proceedings of NASPA, Leadership Exchange, 2003: 12-16.

Baren R., Watson J. Communication Skills Development within the Engineering Curriculum, en: Proceedings of the frontiers in Education Conference, volumen 1, 1991, pp.419-424.

Brown S.L., Eisehhart K.M. Product Development: Past Research, Present Findings and Future Directions. Academy Management Review, volumen 20, 1995: 342-378.

Clarke N. Emotional Intelligence and its Relationship to Transformational Leadership and Key Project Manager Competences. Project Management Journal, volumen 41 (núnero 2), 2010: 5-20.

Cordoba-Wentlin R.M. Human Behavior Skills in Engineering Education. Taymond Price University of Illinois-Urbana Champaign, American Society for Engineering Education (AC 2007-398), 2007.
Crawford A.S. Leadership Education at the University of Michigan, en: Proceedings at the ASEE Annual Conference, 1998, pp. 1-25.

Darling A.L., Daneels D.P. Practicing Engineers Talk About the Importance to Talk: A Report of the Role of Oral Communication in the Workplace. Communication Education, volumen 53, 2003: 1-16.

Dehesa de la G. Some Suggestions from an Economist to a New High-Tech Starter. Engineering a High-Tech Business: Entrepreneural Experiences \& Insights, en: López Higuera José Miguel y Culshaw Brian, eds. SPIE Press, Washington, USA, 2008.

Denison R.D. Cultura corporative, Bogotá, Fondo Editorial LEGIS, 1991.

Dewey J. Moral Principles in Education. Carbondale, IL: Souther Illinois University Press, 1909/1975.

Gilbert M., Cordey-Hayes M. Understanding the Process of Knowledge Transfer to Achieve Successful Technological Innovation. Technovation, volumen 16 (número 6), 1996: 301-312.

Goleman D. What Makes a Leader? IEEE Engineering Management Review, volumen 27 (número 1), 1999: 4-11.

Grigg T, Adopting an Entrepreneurial Approach in Universities. Journal of Engineering Technology \& Management, volumen 11, 1994: 273-298.

Hauser J.R., Zettelmeyer F. Metrics to Evaluate R, D \& E. Research Technology Management, volumen 40 (número 4), 1997: 32-46.

Hecker P.A. Successful Consulting Engineer: A Lifelong Learning. Journal of Management in Engineering, 1997: 62-65.

Herkert J.R. Engineering Ethics Education in USA: Content, Pedagogy and Curriculum. European Journal of Engineering Education, volumen 25, 2000: 303-313.

Hissey T.W. Education and Careers 2000. Enhanced Skills for Engineers. Proceedings of the IEEE, volumen 8, 2000: 1367-1370.

Hsu C. Dilbert's Dilemma. US News and World Report, 2004, pp. 54-59.

Igbaria M., Kassicieh S.K., Silver M. Career Orientations and Career Success Among Research, and Development and Engineering Professionals. J. Eng. Technol. Management, volumen 16 (número 1), 1999: 29-54.

Ignatius A. Modesty, Humility and Humanity. Harvard Bisines Review, volumen 88 (número 6), 2010: 14.

Inceoglu I., Shukla N. Student Engagement in the Context of Work Based Learning as an Unconventional Form of Higher Education. The on Line Journal of New Horizons in Education, volumen 1 (número 1), 2011: 23-31.

Indexmundi México, Tasa de desempleo [en línea] [fecha de consulta: 14 de junio de 2011] Disponible en: http://www.indexmundi.com/es/mexico/tasa_de_desempleo.html.

INEGI Desocupación, Encuesta Nacional de Educación y Empleo; Instituto Nacional de Estadística, Geografía e Informática [en línea] [fecha de consulta: 14 de junio de 2011. Disponible en: http://dgcnesyp.inegi.org.mx/cgi-win/bdiecoy. exe/621?s=est\&c=12933. 
Jennings B. Enlightenment and Enchantment: Technology and Moral Limits. Technology in Society, volumen 32, 2010: 25-30.

Kondo M. Networking for Technology Acquisition and Transfer. International Journal for Technology Management, volumen 32, (numeros 1-2), 2005: 154-175, Inderscience Enterprises Ltd.

Kratzer J., Leenders R.T., Van Engelen J.M.L. The Social Structure of Leadership and Creativity in Engineering Design Teamn: An Empirical Analysis. Journal of Engineering Technology Management, volumen 25, 2008: 269-286.

Lindgard R., Berry E. Teaching Teamwork Skills in Software Engineering Based on an Understanding of Factors Affecting Group Performance, en: Proceedings of the Frontiers in Education Conference, 2002, pp.1-5.

Loui M.C. Engineering Courage: The Social Construction of SelfConfidence in a Course of Professional Ethics. Unpublished Research Study, University of Illinois at Urbana-Champaign, IL, USA, 2004.

Marshall J., Marshall J. Fostering Moral Autonomy of Future Engineers Through Engineering Classrooms. American Society for Engineering Education, Doc. AC 2007-367, 2007.

Nguyen D.Q. The Essential Skills and Attributes of an Engineer: A Comparative Study of Academics, Industry Personnel, and Engineering Students. Global Journal of Engineering Education, volumen 2, 1998: 65-76.

Preston J, Success Factors in Technology Based Entrepreneurship, MIT Entrepreneurship Center, 2001.

Robar T.Y. Communication and Career Advancement. Journal of Management in Engineering, 1998: 26-28.

Scott S.G. Social Identification Effects in Product and Process Development Teams. Journal Engineering Technology Management, volumen 14, 1997: 97-127.

Selinger C. Stuff you don't Learn in Engineering School. IEEE Spectrum, volumen 40, 2003: 49-52.

SEP. Planeación institucional, metodología [en línea], 2009, [fecha de consulta: 02 de agosto de 2012. Disponible en: http:// www.dgb.sep.gob.mx/informacion_academica/planeacion_ institucional/planeacion_inst_v2_090831.pdf.

Sheppard D.S., Macatangay K., Colby A., William W.M. Educating Engineers: Designing for the Future of the Field. The Carniegie Foundation for the Advancement of Teaching, Jossey-Bass, Wiley, 2009.

Simmons M.E. The Essential Interpersonal Skills for Junior Engineers and Engineering Managers, tesis (doctorado), Cincinatti, Ohio, Union Institute, 1999.
Stephan K.D. Is Engineering Ethics Optional? IEEE Technology and Society Magazine, volumen 20, 2002: 6-12.

Sundstrom E., De Meuse,K., Futrell D. Work Teams: Application and Effectiveness. American Psychologist, volumen 45 (número 2), 1990: 20-133.

Sunindijo R., Hadikisumo B. Benefits of Emotional Intelligence to Project Management: Study of Leadership and Conflict Resolution Style of Project Managers in Thayland, en: Proceedings of Research Week International Conference, The Queensland University of Technology, Brisbane, Australia, 2005, pp. 40-52.

Valencia R.D. Crisis y futuro de la ingeniería. Facultad de Ingeniería, Universidad de Antioquia, Colombia. Ingeniería y Sociedad, volumen 1 (número 1), 2010: 1-6.

Widding B., Lohmann J. Educating Engineers for the Global Workplace. Report of the Study: In Search of Global Engineering Excellence: Educating the Next Generation of Engineers for the Global Workplace. American Society for Engineering Education (AC 2007-854), 2007.

Yurtseven H.O. How does the Image of Engineering Affect Student Recruitment and Retention? A Perspective from the USA, en: Proceedings of the 1998 Annual ASEE Conference, 2001.

Zhang Y., Bai L., Zhang D. Strengthening the Engineer's Lifelong Education. Advanced Materials Research, volúmenes 156-157, 2011: 241-244.

\section{Este artículo se cita:}

Citación estilo Chicago

Vega-González, Luis Roberto. La educación en ingeniería en el contexto global: propuesta para la formación de ingenieros en el primer cuarto del Siglo XXI. Ingeniería Investigación y Tecnología, XIV, 02 (2013): 177-190.

\section{Citación estilo ISO 690}

Vega-González L.R. La educación en ingeniería en el contexto global: propuesta para la formación de ingenieros en el primer cuarto del Siglo XXI. Ingeniería Investigación y Tecnología, volumen XIV (número 2), abril-junio 2013: 177-190. 


\section{Semblanza del autor}

Luis Roberto Vega-González. Es ingeniero mecánico electricista por la Facultad de Ingeniería de la UNAM, recibió su maestría en ingeniería en sistemas en 1987 y su maestría en administración de las organizaciones en 2002, ambas por la Universidad Nacional Autónoma de México (UNAM). Desde 1980 ha realizado proyectos de ingeniería para varias firmas de instrumentación industrial atendiendo las industrias del petróleo (PEMEX) y de la energía (CFE). A partir de 1993 ha realizado administración tecnológica de proyectos de investigación aplicada, desarrollo tecnológico e innovación en varias dependencias universitarias. Actualmente es coordinador de vinculación y gestión tecnológica del Centro de Ciencias Aplicadas y Desarrollo Tecnológico (CCADET) de la UNAM, trabajando activamente en proyectos para los sectores de salud, educación, energía y medio ambiente para distintas organizaciones públicas y privadas. 